\title{
A REVIEW OF HUMAN ACTIVITY RECONGNITION AND BEHAVIOR UNDERSTANDING IN VIDEO SURVEILLANCE
}

\author{
A.R.Revathi ${ }^{1}$ and Dhananjay Kumar ${ }^{2}$ \\ ${ }^{1}$ Research Scholar, Department of Information Technology, Anna University, \\ MIT campus \\ revathirajendran2010@gmail.com \\ ${ }^{2}$ Associate Professor, Department of Information Technology, Anna \\ University, MIT campus \\ dhananjayks@gmail.com
}

\begin{abstract}
This paper presents a survey of activity recognition and understanding the behaviour of human activity in video sequence. The major goal of this paper is to provide a general review on the overall process of a surveillance system used in the current trend. Visual surveillance system is directed on automatic identification of events of interest, especially on tracking and classification of moving objects. The processing step of the video surveillance system includes the following stages: Surrounding model, object representation, object tracking, activity recognition and behaviour understanding. It describes techniques that use to define a general set of activities that are applicable to a wide range of scenes and environments in video sequence. The review methods used for real-time surveillance through a set of events for further analysis triggering, including virtual fencing, speed profiling, behaviour classification, anomaly detection, and object interaction.
\end{abstract}

KEYWORDS: Objects Classification, behaviour understanding, Tracking, Data Fusion.

\section{INTRODUCTION}

As an active research topic in computer visions are the dynamic scenes detection, classifying object, tracking and recognizing activity and description of behaviour. Visual surveillance strategies have long been in use to gather information and to monitor people, events and activities. Video surveillance works as to detect moving object [1], [3], [6], [7], classify [8], [10] the detected object track [11], [13] them through the sequence of images and analysis the behaviours. Visual surveillance technologies [22], CCD cameras, thermal cameras and night vision device are the three most widely used devices in the visual surveillance market. The main goal of visual surveillance is not only to monitor, but also to automate the entire surveillance task. The goal of visual surveillance is to develop intelligent visual surveillance to replace the traditional passive video surveillance that is proving in effective as the numbers of cameras exceed the capability of human operators to monitor them. The automated surveillance systems can be implemented for both offline like storing the video sequence and to analyse the information in that sequence. But now days online surveillance system is very much needful in all public and private sectors due to predict and avoid unwanted movements, terrorist activities in those areas. It is helpful for traffic monitoring, transport networks, traffic flow analysis,

Natarajan Meghanathan, et al. (Eds): SIPM, FCST, ITCA, WSE, ACSIT, CS \& IT 06, pp. 375-384, 2012. (C) CS \& IT-CSCP 2012 
understanding of human activity [20], [16], [15], home nursing, monitoring of endangered species, and observation of people and vehicles within a busy environment along many others to prevent theft and robbery. Some of the areas where video surveillance system place a major role in many application are 1) for military security 2)patrolling of country borders 3)extracting statistics for sport activities 4)surveillance of forests for fire detection 5) patrolling of highways and railway for accident detection.

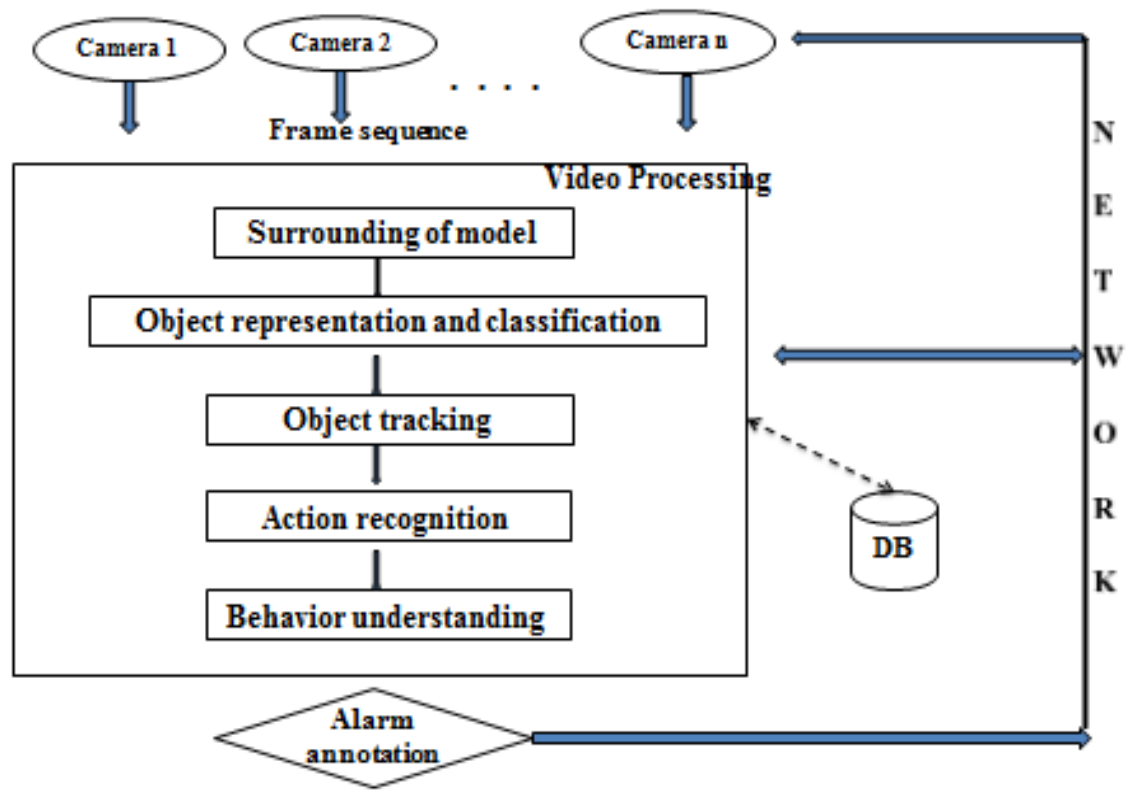

Figure 1: Overview of visual surveillance.

In a video stream monitoring or analysing suspected anomalies can be divided in to three categories, which are single person with single object interaction, multiple people with multiple objects, and group behaviour[18], [13]. This paper focused to visual surveillance in the direction of surrounding of model, objects segmentation, classification, tracking and finally reached to behaviour understanding and action recognition. Many papers have been introducing various new concepts in this way. This paper reviews general strategies of various levels involved in video surveillance and analysis to challenges and feasibilities for combining object tracking, motion analysis, behaviour analysis for human subject understanding and behaviour understanding. The inspiration of writing and survey paper on this topic for a domain specific application is to review and gain insight in video surveillance. Surrounding of model includes object detection and object segmentation. It could be carried out by different approaches includes frame to frame difference, background subtraction and motion analysis using optical flow techniques. This becomes very popular due to robustness to noise, shadow and changing of light conditions.

\section{SURROUNDING OF MODEL}

Surrounding modelling is also known as Background modelling .It is currently used to detect moving objects in video acquired from static cameras. Numerous statistical methods have been developed over the recent years. The aim of this paper is to provide an extended and updated 
survey of the recent [23], [24], [25] researches which concern statistical background modelling. Murshed, $\mathrm{M}$ was proposed an edge segment based statistical background modelling [1] algorithm and a moving edge detection framework for the detection of moving objects. This paper actually focused about various methods of background modelling like traditional pixel based, edge pixel based and edge segment based approaches. He proposed this background modelling for natural image sequence with presence of illumination variation and noise. Yun Chu Zhang was analysed the background mechanism using GMM [2] model. Here this model updates new strategy which weighs the model adaptability and motion segmentation accuracy. But these works not focus dynamic moves in frame sequence. Wei Zhou [5] was proposed the dynamic background subtraction using spatial colour binary patterns. In addition to a refined model is designed to refine contour of moving objects. This method improves the accuracy of subtracting and detecting moving objects in dynamic scenes with presence of data driven model. Richard J.Radke [23] has written survey about image change detection algorithm with several challenge issues with solved by Stauffer and Grimson's background modelling with real times image. ViBe: A Universal Background Subtraction Algorithm [6] for Video Sequences paper presence a technique for motion detection which stores a set of value taken in the past in the same location or in the neighbourhood. It then compares this set to current pixel value in order to determine whether the pixel belongs to the background and to adopt the model which substitutes from the background model.

Table 1: Summary of surrounding model

\begin{tabular}{|c|l|l|}
\hline Year & \multicolumn{1}{|c|}{ Author } & \multicolumn{1}{c|}{ Concepts } \\
\hline 2010 & $\begin{array}{l}\text { Murshed, M, Ramirez. A, } \\
\text { Chae. O }\end{array}$ & $\begin{array}{l}\text { An Edge Segment Based statistical } \\
\text { background modeling algorithm [1]. }\end{array}$ \\
\hline 2011 & $\begin{array}{l}\text { Yun Chu Zhang, Ru Min Zhang, } \\
\text { Shi Jun Song }\end{array}$ & $\begin{array}{l}\text { Research on GMM Background Modeling } \\
\text { and Covariance Estimation [2] }\end{array}$ \\
\hline 2011 & $\begin{array}{l}\text { Barnich.O, Van } \\
\text { Droogenbroeck.M }\end{array}$ & $\begin{array}{l}\text { ViBe: A Universal Background } \\
\text { Subtraction Algorithm for Video } \\
\text { Sequences[6] }\end{array}$ \\
\hline
\end{tabular}

\section{OBJECT REPRESENTATION AND CLASSIFICATION}

Object classification is a standard pattern recognition task. To track objects and analyse the behaviour, it is essential to correctly classify moving objects. There are two different categories of approaches for classifying moving objects like, shape based and motion based classification. Lun Zhang [7] has introduced a novel method for real time robust object classification in diverse camera viewing angles by applying appearance based techniques. A new descriptor, the multi block local binary pattern is used to capture the large scale structure in object appearance. An adaboost algorithm is introduced to select a subset of selected features as well as construct a different classifier for classification. However the above method has a overhead of the real time classification in the automated surveillance. To overcome real time classification, Hota, R.N [8] has proposed a online feature selection method which gives a good subset of features while the machine learns the classification task and use these selected features for object classification.

Table 2: Summary of object representation and classification

\begin{tabular}{|c|l|l|}
\hline Year & \multicolumn{1}{|c|}{ Author } & \multicolumn{1}{c|}{ Concept } \\
\hline 2007 & $\begin{array}{l}\text { Lun Zhang, Li S.Z, } \\
\text { Xiaotong Yuan, Shiming } \\
\text { Xiang }\end{array}$ & $\begin{array}{l}\text { Multi-block local binary pattern (MB-LBP), is } \\
\text { proposed to capture the large-scale structures in } \\
\text { object appearances [7]. }\end{array}$ \\
\hline 2007 & Hota, R.N. Venkoparao, & $\begin{array}{l}\text { Shape Based Object Classification for } \\
\text { Automated Video Surveillance with Feature }\end{array}$ \\
\hline
\end{tabular}




\begin{tabular}{|l|l|l|}
\hline & V. Rajagopal, A & Selection [8]. \\
\hline 2012 & Robert Sorschag & $\begin{array}{l}\text { A Flexible Object-of-Interest Annotation } \\
\text { Framework for Online Video Portals. To } \\
\text { present an automatic selection approach to } \\
\text { support the use of different recognition } \\
\text { strategies for different objects [9]. }\end{array}$ \\
\hline
\end{tabular}

All the above methods have very little accuracy in the multi class objects. Yaniv Gurwicz [31] has proposed a Multiclass object classification for real-time video surveillance systems which introduce an approach for classifying objects in both low and high resolution images in real world scenarios. In this method have several features that jointly leverage the divisions between various classes and also provide a feature selection procedure based on the entropy gain. Robert Sorschag [9] has proposed a Flexible object-of-interest Annotation framework for online video portals using content based analysis method. This framework slightly varies from other content based analysis method, since it possesses some outstanding features that offer good prospects for real video portal applications.

\section{OBJECT TRACKING}

The objective of video tracking is to associate target objects in consecutive video frames. The association can be especially difficult when the objects are moving fast relative to the frame rate. Blob tracking, kernel-based tracking, Contour tracking are some common target representation and localization algorithms. Ruolin Zhang [32] has proposed adaptive background subtraction about the video detecting and tracking moving object. He use median filter to achieve the background subtraction. This algorithm is used for both detecting and tracking moving objects in sequence of video. This algorithm never support for multi feature based object detection. Hong $\mathrm{Lu}$ and Hong Sheng $\mathrm{Li}[10]$ were introduced a new approach to detect and track the moving object. The affine motion model and the non-parameter distribution model are utilized to represent the object and then the motion region of the object is detected by background difference while Kalman filter estimating its affine motion in next frame. The author shows Experimental results and proof the new method can successfully track the object under such case as merging, splitting, scale variation and scene noise. The author Bayan [11] talks about adaptive mean shift for automated multi tracking. Mixture of Gaussian model extracted Foreground and then followed by shadow and noise removal to initialise the object trackers and also to make the system more efficient by decreasing the search area and the number of iterations to converge for the new location of the object. Trackers are automatically relaxed to solve the potential problems that may occur because of the changes in objects' size, shape, to handle occlusion-split between the tracked objects and to detect newly emerging objects as well as objects that leave the scene. Using a shadow removal method has increases the tracking accuracy.

Table 3: Summary of object tracking

\begin{tabular}{|l|l|l|}
\hline Year & \multicolumn{1}{|c|}{ Author } & \multicolumn{1}{c|}{ Concept } \\
\hline 2011 & $\begin{array}{l}\text { Hong Lu, Hong Sheng Li, } \\
\text { Lin Chai, Shu Min Fei, } \\
\text { Guang Yun Liu }\end{array}$ & $\begin{array}{l}\text { Multi-Feature Fusion Based Object Detecting } \\
\text { and Tracking. Then the motion region of the } \\
\text { object is detected by background difference } \\
\text { while Kalman filter estimating its affine motion } \\
\text { in next frame [10]. }\end{array}$ \\
\hline 2012 & Beyan, C. Temizel, A & $\begin{array}{l}\text { Adaptive mean-shift for automated multi object } \\
\text { tracking [11]. }\end{array}$ \\
\hline
\end{tabular}




\begin{tabular}{|l|l|l|}
\hline 2012 & Jiu Xu, Chenyuan Zhang & $\begin{array}{l}\text { Modified codebook foreground detection and } \\
\text { particle filter [12]. }\end{array}$ \\
\hline 2011 & $\begin{array}{l}\text { Blanco Adán, Carlos } \\
\text { Roberto del, Jaureguizar } \\
\text { Nuñez, Fernando, García } \\
\text { Santos }\end{array}$ & $\begin{array}{l}\text { Bayesian Visual Surveillance, a Model for } \\
\text { Detecting and Tracking a variable number of } \\
\text { moving objects [13]. }\end{array}$ \\
\hline
\end{tabular}

Jiu $\mathrm{Xu}$ [12] was proposed the foreground object detection process by the modified code book. The modified codebook foreground detection and particle filter are used for object tracking by detection for surveillance system. This code book was created by adding the orientation and magnitude of the block gradient, the codebook model contains not only information of color, but also the texture feature in order to further reduce noises and refine more entire foreground regions using modified code book. By calculate the local binary pattern of the edge of the foreground objects which could have a good performance in describing the shape and texture of the objects. But this will give only solution for one or two object with particle occlusion. Bayesian Visual Surveillance [13], a Model for Detecting and Tracking a variable number of moving objects paper was focus for many objects with occlusion. Video object detectors generate an unordered set of noisy, false, missing, split, and merged measurements that make extremely complex the tracking task. Blanco Adán [13] was introduced a Bayesian Visual Surveillance Model is proposed that is able to manage undesirable measurements. Particularly, split and merged measurements are explicitly modelled by stochastic processes.

\section{DESCRIPTION AND BEHAVIOUR UNDERSTANDING}

Human action recognition is a challenging zone of research because of its various potential applications in visual surveillance. Automatic human activity recognition in video using background modelling and spatio-temporal template matching based technique [28] can be proposed by Chandra Mani Sharm. This activity recognition processed based on the spatiotemporal template matching. The Motion history images are used to construct the Spatio-temporal templates and object shape information for different human activities in a video like walking, standing, bending, sleeping and jumping. From figure 2, the results show that method can determine the three activities walking, bending, and standing accurately. The location of objects in bending and standing activities remains still whereas location of human object in walking position changes with respect to time from frame 1 to 275 . But the proposed method accurately measures these activities.

Table 4: Description and behaviour understanding

\begin{tabular}{|c|c|c|}
\hline Year & Author & Concept \\
\hline 2011 & $\begin{array}{l}\text { Chandra Mani Sharma, } \\
\text { Alok Kr. Singh } \\
\text { Kushwaha, Swati } \\
\text { Nigam, Ashish Khare }\end{array}$ & $\begin{array}{l}\text { Automatic human activity recognition in video using } \\
\text { background modeling and spatio-temporal template } \\
\text { matching based technique [28]. }\end{array}$ \\
\hline 2011 & $\begin{array}{l}\text { Chun-hao Wang, } \\
\text { Yongjin Wang, Ling } \\
\text { Guan }\end{array}$ & $\begin{array}{l}\text { Event detection and recognition using histogram of } \\
\text { oriented gradients and hidden markov models [27]. }\end{array}$ \\
\hline 2008 & $\begin{array}{l}\text { Tao Xiang } \quad \text { and } \\
\text { Shaogang Gong }\end{array}$ & $\begin{array}{l}\text { Online LRT based behaviour recognition approach is } \\
\text { advantageous over the commonly used Maximum } \\
\text { Likelihood (ML) method in differentiating ambiguities } \\
\text { among different behaviour classes observed online [30]. }\end{array}$ \\
\hline
\end{tabular}


For health care, to establish new healthcare systems to ensure the safety of elderly people at home. A new method is proposed to detect falls by analysing human shape deformation during a video sequence. A shape matching technique is used to track the person's silhouette along the video sequence. Finally, falls are detected from normal activities using a Gaussian mixture model. Chun-hao Wang [27] presents an approach for object detection and action recognition in video surveillance scenarios. This system utilizes a Histogram of Oriented Gradients (HOG) method for object detection, and a Hidden Markov Model (HMM) for capturing the temporal structure of the features. Decision making is based on the understanding of objects motion trajectory and the relationships between objects' movement and events. David Nicholas Olivieri proposed[29] such software based upon a spatio-temporal motion representation, called Motion Vector Flow Instance (MVFI) templates that capture relevant velocity information by extracting the dense optical flow from video sequences of human actions. Tao Xiang[30] was proposed novel framework is developed for automatic behaviour profiling and online anomaly sampling/detection without any manual labelling of the training data set. The framework consist various key components: A compact and effective behaviour representation method is developed based on discrete-scene event detection. The similarity between behaviour patterns are measured based on modelling each pattern using a Dynamic Bayesian Network (DBN). The natural grouping of behaviour patterns is discovered through a novel spectral clustering algorithm with unsupervised model selection and feature selection on the eigenvectors of a normalized affinity matrix. A composite generative behaviour model is constructed that is capable of generalizing from a small training set to accommodate variations in unseen normal behaviour patterns. A runtime accumulative anomaly measure is introduced to detect abnormal behaviour, whereas normal behaviour patterns are recognized when sufficient visual evidence has become available based on an online Likelihood Ratio Test (LRT) method. Ali S.F [20] has given a complete framework and approach for making the system of Automatic Target Recognition System we suggest the name of IVATRs (Intelligent Video Automatic Target Recognition System). This framework will be helpful for making a bridge between the theoretical models of AI techniques and their implementations with hardware.

\section{DATA FUSION}

Data fusion is important for occlusion handling and continuous tracking. Occlusions remove the direct correspondence between visible areas of objects and the objects themselves by introducing ambiguity in the interpretation of the shape of the occluded object. Dockstader et al. [18] use a Bayesian network to fuse 2-D state vectors acquired from various image sequences to obtain a 3D state vector. Collins et al. [19] design an algorithm that obtains an integrated representation of an entire scene by fusing information from every camera into a 3-D geometric coordinate system. Kettnaker et al. [17] synthesize the tracking results of different cameras to obtain an integrated trajectory.

Table 5: Summary of widely used techniques for visual surveillance

\begin{tabular}{|c|l|l|}
\hline Year & \multicolumn{1}{|c|}{ Author } & \multicolumn{1}{c|}{ Concepts } \\
\hline 2007 & $\begin{array}{l}\text { Lun Zhang, Li S.Z, Xiaotong } \\
\text { Yuan, Shiming Xiang }\end{array}$ & $\begin{array}{l}\text { Object Classification in Video Surveillance Based } \\
\text { on Appearance Learning [7]. }\end{array}$ \\
\hline 2007 & $\begin{array}{l}\text { Hota.R.N. Venkoparao.V } \\
\text { Rajagopal.A }\end{array}$ & $\begin{array}{l}\text { Shape Based Object Classification for Automated } \\
\text { Video Surveillance with Feature Selection [8]. }\end{array}$ \\
\hline 2008 & Tao Xiang and Shaogang Gong & $\begin{array}{l}\text { Video Behavior Profiling for Anomaly Detection } \\
\text { [30] }\end{array}$ \\
\hline
\end{tabular}




\begin{tabular}{|l|l|l|}
\hline 2010 & $\begin{array}{l}\text { Murshed, M, Ramirez. A, } \\
\text { Chae. O }\end{array}$ & $\begin{array}{l}\text { An Edge Segment Based Moving Object Detection } \\
\text { Approach [1]. }\end{array}$ \\
\hline 2011 & $\begin{array}{l}\text { Yun Chu Zhang, Ru Min } \\
\text { Zhang, Shi Jun Song }\end{array}$ & $\begin{array}{l}\text { Research on GMM Background Modeling and } \\
\text { Covariance Estimation [2]. }\end{array}$ \\
\hline 2011 & Barnich.O,Van roogenbroeck.M & $\begin{array}{l}\text { ViBe: A Universal Background Subtraction } \\
\text { Algorithm for Video Sequences [6]. }\end{array}$ \\
\hline 2011 & $\begin{array}{l}\text { Hong Lu, Hong Sheng Li, Lin } \\
\text { Chai, Shu Min Fei, Guang Yun } \\
\text { Liu }\end{array}$ & $\begin{array}{l}\text { Multi-Feature Fusion Based Object Detecting and } \\
\text { Tracking [10]. }\end{array}$ \\
\hline 2011 & $\begin{array}{l}\text { Blanco Adán, Carlos Roberto } \\
\text { del, Jaureguizar Nuñez, } \\
\text { Fernando, García Santos }\end{array}$ & $\begin{array}{l}\text { Bayesian Visual Surveillance, a Model for Detecting } \\
\text { and Tracking a variable number of moving objects } \\
\text { [13]. }\end{array}$ \\
\hline 2011 & $\begin{array}{l}\text { Chandra Mani Sharma, Alok } \\
\text { Kr. Singh Kushwaha, Swati } \\
\text { Nigam, Ashish Khare }\end{array}$ & $\begin{array}{l}\text { Automatic human activity recognition in video using } \\
\text { background modeling and spatio-temporal template } \\
\text { matching based technique [28]. }\end{array}$ \\
\hline 2011 & $\begin{array}{l}\text { Chun-hao Wang, Yongjin } \\
\text { Wang, Ling Guan }\end{array}$ & $\begin{array}{l}\text { Event detection and recognition using histogram of } \\
\text { oriented gradients and hidden markov models [27]. }\end{array}$ \\
\hline 2012 & $\begin{array}{l}\text { Robert Sorschag } \\
\text { A Flexible Object-of-Interest Annotation } \\
\text { Framework for Online Video Portals [9]. }\end{array}$ \\
\hline 2012 & Beyan, C. Temizel, A & $\begin{array}{l}\text { Adaptive mean-shift for automated multi object } \\
\text { tracking [11]. } \\
\text { Modified codebook foreground detection and } \\
\text { particle filter [12]. }\end{array}$ \\
\hline 2012 & Jiu Xu, Chenyuan Zhang & \multicolumn{1}{|c|}{ and } \\
\hline
\end{tabular}

\section{CONCLUSION}

Most current automated video surveillance systems can process video sequence and perform almost all key low-level functions, such as motion detection and segmentation, object tracking, and object classification with good accuracy. But technical interest in video surveillance has moved from such low-level functions to more complex scene analysis to detect human and/or other object behaviors, i.e., patterns of activities or events, for standoff threat detection and prevention. Existing behavior analysis systems focus on the predefined behaviors, e.g., to combine the results of an automated video surveillance system with spatiotemporal reasoning about each object relative to the key background regions and other objects in the scene. Advanced behavior analysis systems have begun to exploit the capability to automatically capture and learn new behaviors by pattern matching, and further present the behavior to the specialists for confirmation. This paper reviews and exploits developments and general strategies of stages involved in video surveillance and analyses the challenges and feasibility for combining object tracking, motion analysis, behavior analysis, and biometrics for stand-off human subject identification and behavior understanding. Behavior understanding and activity recognition using visual surveillance involves the most advanced and complex researches in image processing, computer vision, and artificial intelligence. There were many diverse methods have been used while approaching this challenge; and they varied and depended on the required speed, the scope of application, and resource availability, etc. 


\section{REFERENCES}

[1]. M. Murshed, A.Ramirez, O.Chae, (2010) "Statistical Background Modelling: An Edge Segment Based Moving Object Detection Approach" IEEE International Conference on Advanced Video and Signal Based Surveillance (AVSS).

[2]. Yun Chu Zhang, Ru Min Zhang, Shi Jun Song, (2011) "Research on GMM Background Modelling and its Covariance Estimation” Journal on Advanced Materials Research (Volumes 383 - 390).

[3] Peng Chena, Xiang Chenb, Beibei Jina, Xiangbing Zhua (2012) “Online EM Algorithm for Background Subtraction".

[4] K. Mahesh, K. Kuppusamy, (2012) "Video Segmentation using Hybrid Segmentation Method", European Journal of Scientific Research, ISSN 1450-216X Vol.71 No.3, pp. 312-326.

[5] Wei Zhou; Yu Liu; Weiming Zhang; Liansheng Zhuang; Nenghai Yu, (2011) "Dynamic Background Subtraction Using Spatial-Color Binary Patterns", International Conference on Image and Graphics (ICIG).

[6] Barnich.O, Van Droogenbroeck.M,(2011) “ViBe: A Universal Background Subtraction Algorithm for Video Sequences” IEEE Transactions on Image Processing, Vol 20,No.6.

[7] Lun Zhang, Li S.Z, Xiaotong Yuan, Shiming Xiang, (2007) "Real-time Object Classification in Video Surveillance Based on Appearance Learning" IEEE Conference on Computer Vision and Pattern Recognition.

[8] R.N Hota, V.Venkoparao, A.Rajagopal, (2007) "Shape Based Object Classification for Automated Video Surveillance with Feature Selection”, 10th International Conference on Information Technology, ICIT.

[9] Robert Sorschag, (2012) “A Flexible Object-of-Interest Annotation Framework for Online Video Portals" article on Visual Information Retrieval.

[10] Hong Lu, Hong Sheng Li, Lin Chai, Shu Min Fei, Guang Yun Liu, (2011) "Multi-Feature Fusion Based Object Detecting and Tracking”, journal on Applied Mechanics and Materials.

[11] C. Beyan, A Temizel, (2012), “Adaptive mean-shift for automated multi object tracking” IET on Computer Vision.

[12] Jiu Xu, Chenyuan Zhang, (2012) "Object tracking by detection for video surveillance systems based on modified codebook foreground detection and particle filter" International conference on "Intelligent Signal Processing and Communications Systems (ISPACS)”.

[13] Blanco Adán, Carlos Roberto del and Jaureguizar Nuñez, Fernando and García Santos,(2011) "Bayesian Visual Surveillance, a Model for Detecting and Tracking a variable number of moving objects", IEEE International Conference on Image Processing, 13-14 de Enero del 2011.

[14] Caroline Rougier, Jean Meunier, Alain St-Arnaud, and Jacqueline Rousseau,(2011) "Robust Video Surveillance for Fall Detection Based on Human Shape Deformation” IEEE Transaction on circuits and systems for video technology, vol 21, no 5.

[15] NeilM Robertson and IanD Reid, (2011) “Automatic Reasoning about Causal Events in Surveillance Video", EURASIP Journal on Image and Video Processing .

[16] Jenq-Neng Hwang, (2011) "Keynote talk 4: Automated understanding of video object events in a distributed smart camera network", International Conference on Advanced Technologies for Communications (ATC). 
[17] V. Kettnaker and R. Zabih,(1999) "Bayesian multi-camera surveillance," in Proc. IEEE Conf. Computer Vision and Pattern Recognition, pp. 253-259.

[18] S. L. Dockstader and A. M. Tekalp, (2001) "Multiple camera tracking of interacting and occluded human motion,” Proc. IEEE, vol. 89, pp. 1441-1455.

[19] R. T. Collins, A. J. Lipton, H. Fujiyoshi, and T. Kanade,(2001) "Algorithms for cooperative multisensor surveillance,” Proc. IEEE, vol. 89, pp. 1456-1477.

[20] Ali, S.F.; Jaffar, J.; Malik, A.S.,(2012) "Proposed framework of Intelligent Video Automatic Target Recognition System (IVATRs)”, National Postgraduate Conference (NPC).

[21] G. Jing, C.E. Siong and D. Rajan,(2004) "Foreground motion detection by difference-based spatial temporal entropy image," IEEE Region 10 Conference Proceedings: Analog and Digital Techniques in Electrical Engineering, pp. 379-382.

[22] Arun Hampapyr, Lisa Brown, Jonathan Connell, Ahmet Ekin, Norman Haas,(2005) "Smart Video Surveillance Exploring the concept of multiscale spatiotemporal tracking", IEEE Signal Processing magazine, pp. 38-50.

[23] S. Radke, O.Andra, Al-Kofahi, and B. Roysam,(2005) "Image change detection algorithms: A systematic survey,” IEEE Trans. Image Process., vol. 14, pp. 294-307.

[24] Y. Benezeth, P. Jodoin, B. Emile, H. Laurent, and C. Rosenberger,(2008) "Review and evaluation of commonly-implemented background subtraction algorithms," in Proc. IEEE Int. Conf. Pattern Recognit., pp. 1-4.

[25] S. Elhabian, K. El-Sayed, and S. Ahmed, (2008) "Moving object detection in spatial domain using background removal techniques-State-of-art,” Recent Pat. Computer. Sci., vol. 1, pp. 32-54.

[26] Xin Zhang, Qingming Ya, Kunfeng Wang,(2011) "Hierarchical and Modular Surveillance Systems in ITS” IEEE intelligent systems, IEEE Computer society.

[27] Chun-hao Wang, Yongjin Wang, Ling Guan,(2011) "Event detection and recognition using histogram of oriented gradients and hidden markov models", ACM digital library, ICIAR'11 Proceedings of the 8th international conference on Image analysis and recognition - Volume Part I, Pages 436-445.

[28] Chandra Mani Sharma, Alok Kr. Singh Kushwaha, Swati Nigam, Ashish Khare,(2011) "Automatic human activity recognition in video using background modeling and spatio-temporal template matching based technique",ACM transaction, pp. 97-101.

[29] David Nicholas Olivieri, Iván Gómez Conde, Xosé Antón Vila Sobrino,(2011) "Eigenspace-based fall detection and activity recognition from motion templates and machine learning", journal in Expert Systems with Applications - Elsevier.

[30] Tao Xiang and Shaogang Gong,(2008) "Video Behavior Profiling for Anomaly Detection", IEEE Transactions on Pattern Analysis and Machine Intelligence archive, Volume 30 Issue 5, Pages 893-908.

[31] Yaniv Gurwicz, Raanan Yehezkel, Boaz Lachover,(2011) "Multiclass object classification for realtime video surveillance systems”, Video Analytics Group, NICE Systems Ltd.

[32] Ruolin Zhang, Jian Ding,(2012) "Object Tracking and Detecting Based on Adaptive Background Subtraction”, Stevens Institute of Technology, Hoboken. 


\section{Authors}

A.R.Revathi received the Master of Technology in Computer Science and Engineering from SASTRA University in 2002 and received Bachelor of Engineering in Electronic and Communication and Engineering from Bharathidasan University in 2000.She is currently working as Assistant Professor in Department of Information Technology at SRM Valliammai Engineering College. Her research interests are mainly focused on motion detection, human detection and recognition, and computer vision.

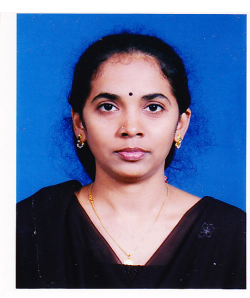

Dr.Dhananjay Kumar received Doctor of Philosophy in Information \& Communication Engineering from Anna University in 2009. He received the Master of Engineering in Industrial Electronics Engineering from Baroda University in 1999 and Master of Technology in Communication Engineering from Pondicherry University in 2001. His primary job at the department of Information Technology, Anna University Chennai involves teaching and research. His technical interest includes mobile computing \& communication, multimedia systems, and signal processing.

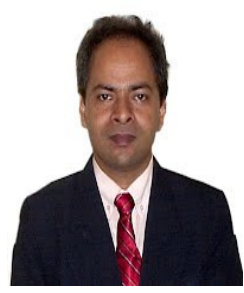

